in I, Abb. 8 dargestellt haben. Allerdings befinden sich bereits im Inneren dieses Dodekaeders die B-Ebenen $\{111\}$, deren Fourier-Koeffizient zwar nicht verschwindet, aber doch kleiner ist als der $\mathrm{zu}$ den Ebenen $\{220\}$ gehörige. Diese Ebenen schneiden die Dodekaederkanten sowohl in der Mitte als auch in der Umgebung der Punkte vom Typ (400) und bringen damit eine Modifizierung des Energielückenverlaufs von $\Gamma_{c}{ }^{\prime \prime}$ gemäß $(\mathrm{I}, 4)$. Wir wollen von diesen Einflüssen jetzt absehen und behalten uns vor, bei`späterer Gelegenheit darauf zurückzukommen, bzw. überhaupt die genaueren Eigenfunktionen anderer Autoren zu benützen (siehe etwa Her$\left.\operatorname{man}^{9,10}\right)$. Der Einfluß solcher Verfeinerungen auf die Durchschlagsformeln bleibt so lange geringfügig, als die Flächen der kleinsten Energielücke im Wellenzahlraum parallel zur Richtung [220] liegen.

Die Anwendung der Gln. (35), (36), (37) liefert die folgenden numerischen Formeln

$$
\begin{aligned}
& F=24,7 I^{3 / 2}\left(22,72+4 \log F-\log I^{3 / 2}\right)^{-1}, \\
& F=17,5 I^{3 / 2}\left(22,38+4 \log F-\log I^{2 / 2}\right)^{-1}, \\
& F=21,4 I^{3 / 2}\left(22,38+4 \log F-\log I^{3 / 2}\right)^{-1} .
\end{aligned}
$$

Die Energielücken betragen für Si $1,11 \mathrm{eV}$ und für Ge 0,76 eV. Damit erhält man

${ }^{9}$ F. Herman, Phys. Rev. 88, 1210 [1952]. für $S i$ :

$F_{[100]}=1,26 ; F_{[110]}=0,92 ; F_{[111]}=1,11 ;$

für $G e$ :

$F_{[100]}=0,68 ; F_{[110]}=0,50 ; F_{[111]}=0,60$.

Das Verhältnis ergibt sich in beiden Fällen zu

$$
F_{[100]}: F_{[110]}: F_{[111]}=1,36: 1,00: 1,20 .
$$

Auch hier ergeben sich gegenüber der Näherungsformel (19) merkliche, wenn auch nicht sehr bedeutende Korrekturen.

Die Absolutwerte der errechneten Durchschlagsfeldstärken sind vor allem deswegen mit einer gewissen Unsicherheit behaftet, weil die effektiven Massen der verschiedenen Materialien nur z.Tl. und nicht sehr genau bekannt sind. Bei unseren numerischen Beispielen wurde stets die effektive Masse gleich der Masse des freien Elektrons gesetzt. - Die Richtungsabhängigkeit der Durchschlagsfeldstärke auf der anderen Seite hängt ganz wesentlich von der Zonenstruktur des Wellenzahlraumes ab. Deshalb könnte man aus Messungen der Richtungsabhängigkeit Rückschlüsse auf die Zonenstruktur ziehen.

${ }^{10}$ F. Herman u. J. Callaway, Phys. Rev. 89, 518 [1953]

\title{
Die spezifische Wärme des flüssigen Schwefels
}

\begin{abstract}
Von H. Braune und O. Möller
Aus dem Institut für Physikalische Chemie der Technischen Hochschule Hannover (Z. Naturforschg. 9a, 210-217 [1954]; eingegangen am 2. November 1953)

Mit Hilfe eines adiabatischen Kalorimeters wurden erstmals die wahren spezifischen Wärmen des flüssigen Schwefels von 120 bis $420^{\circ} \mathrm{C}$ gemessen; aus den Ergebnissen wurden die Wärmeinhalte berechnet, die mit früheren Angaben verglichen wurden. Der Temperaturverlauf der spezifischen Wärme läßt sich mit großer Wahrscheinlichkeit nicht auf Grund der Annahme eines Gleichgewichts $S_{\lambda} \rightleftarrows S_{\mu}$ deuten, sondern man wird wohl Gebrauch machen müssen von der durch den Viskositätsverlauf nahegelegten Hypothese, daß im flüssigen Schwefel Polymerisation eintritt, wobei Menge und Länge der Ketten eine Funktion der Temperatur sind.
\end{abstract}

$\mathrm{D}$ ie spezifische Wärme des flüssigen Schwefels, die sowohl für thermodynamische Rechnungen wie für die Beurteilung der in ihm herrschenden inneren Gleichgewichte von Bedeutung ist, war bis-

1 C. C. Person, C. R. Acad. Sci., Paris 23, 162 [1846]; Ann. chim. phys. (3) 27, 252 [1849]; Ann. Phys., Lpz. 70, 300 [1847].

2 J. Classen, Z. Instrumentenkunde 11, 301 [1891]. her nur recht ungenau bekannt. Einige ältere, z. Tl. stark voneinander abweichende Angaben über mittlere spezifische Wärmen liegen von Person ${ }^{1}$, Clas sen $^{2}$, Jitaka ${ }^{3}$ und Dussy ${ }^{4}$ vor. Lewis und

${ }^{3}$ J. Jitaka, Sci. Rep. Tôhoku Imp. Univ. Ser. I 88, 99 [1919].

4 J. Dussy, C. R. Acad. Sci. Paris 123, 305 [1896]. 
Randall ${ }^{5}$ haben, gestützt auf Messungen des Wärmeinhaltes bei verschiedenen Temperaturen, versucht, unter Zugrundelegung der von Smith $^{6}$ und Mitarbeitern bestimmten Gleichgewichte $S_{\lambda} \rightleftarrows S_{\mu}$ den Temperaturverlauf der wahren spezifischen Wärme zu berechnen. Mondain-Mon$\mathrm{val}^{7}$, der ebenfalls Wärmeinhaltsmessungen ausführte, kommt zu wesentlich anderen Angaben über die spezifischen Wärmen der Schwefelschmelzen. Es erschien uns daher lohnend, das anomale Verhalten der spezifischen Wärme des flüssigen Schwefels durch direkte kalorimetrische Bestimmung der wahren spezifischen Wärme genauer zu untersuchen.

Zur Durchführung der Messungen zwischen 120 und $440^{\circ} \mathrm{C}$ diente uns ein adiabatisch arbeitendes Kalorimeter, für dessen Konstruktion wir manche Anregung einer von Moser ${ }^{8}$ angegebenen Ausführung entnahmen.

\section{Experimenteller Teil}

\section{Versuchsanordnung}

Das Kalorimeter (Abb. 1), das den Schwefel in fester Form aufnimmt, befindet sich im Innern eines etwa $70 \mathrm{~cm}$ langen elektrischen Ofens, dessen Temperaturanstieg bis etwa $500^{\circ} \mathrm{C}$ reguliert werden kann. Ein Kupferbecher mit einer Wandstärke von $6 \mathrm{~mm}$ im Mittelteil des Ofens dient einem guten Temperaturausgleich in der Umgebung des eigentlichen Kalorimeters. Das Kalorimeter befindet sich im Innern eines Glasrohres (55 mm Durchmesser) mit Schliff aus Jenaer Glas, das bis auf etwa 15 Torr evakuiert werden kann. Der Kopf der ganzen Anordnung wird durch eine Stativklemme an einem Eisengerüst gehalten und kann so weit hochgefahren werden, daß das Kalorimeter zugänglich wird.

Den Kalorimetereinsatz aus Jenaer Glas (etwa 85 ccm Inhalt) mit einem eng anliegenden Silberhohlzylinder $(0,5 \mathrm{~mm}$ Wandstärke) umgibt nach Abb. 2 in einem Abstand von $6 \mathrm{~mm}$ ein zweiter Silberhohlzylinder von $1 \mathrm{~mm}$ Wandstärke, der eine vom Ofen bestimmte, in seiner ganzen Ausdehnung konstante ,Umgebungstemperatur" besitzt. Um die Zylinder montieren zu können, sind sie im unteren Drittel aufgeschnitten und mit Gewinden versehen. Ihren konzentrischen Sitz gewährleisten der Schaft und die nach unten auslaufende Spitze des Kalorimetereinsatzes; den Abstand nach oben und unten hält ein Glasring.

Um die Temperatur der beiden Silberhohlzylinder messen zu können, befinden sich auf der Außenseite

5 G. N. Lewis u. M. Randall, J. Amer. Chem. Soc. 33, 476 [1911].

${ }_{6}$ A. Smith u. W. B. Holmes, Z. phys. Chem. A 42, 469 [1903], 54, 257 [1906]; A. Schmith u. C. M. Carson, Z. phys. Chem. A 57, 685 [1907], 77, 661 [1911]. des inneren Zylinders in symmetrischer Anordnung (in Achsenrichtung gesehen um $120^{\circ}$ gegeneinander versetzt) drei, auf der Innenseite des äußeren zwei um

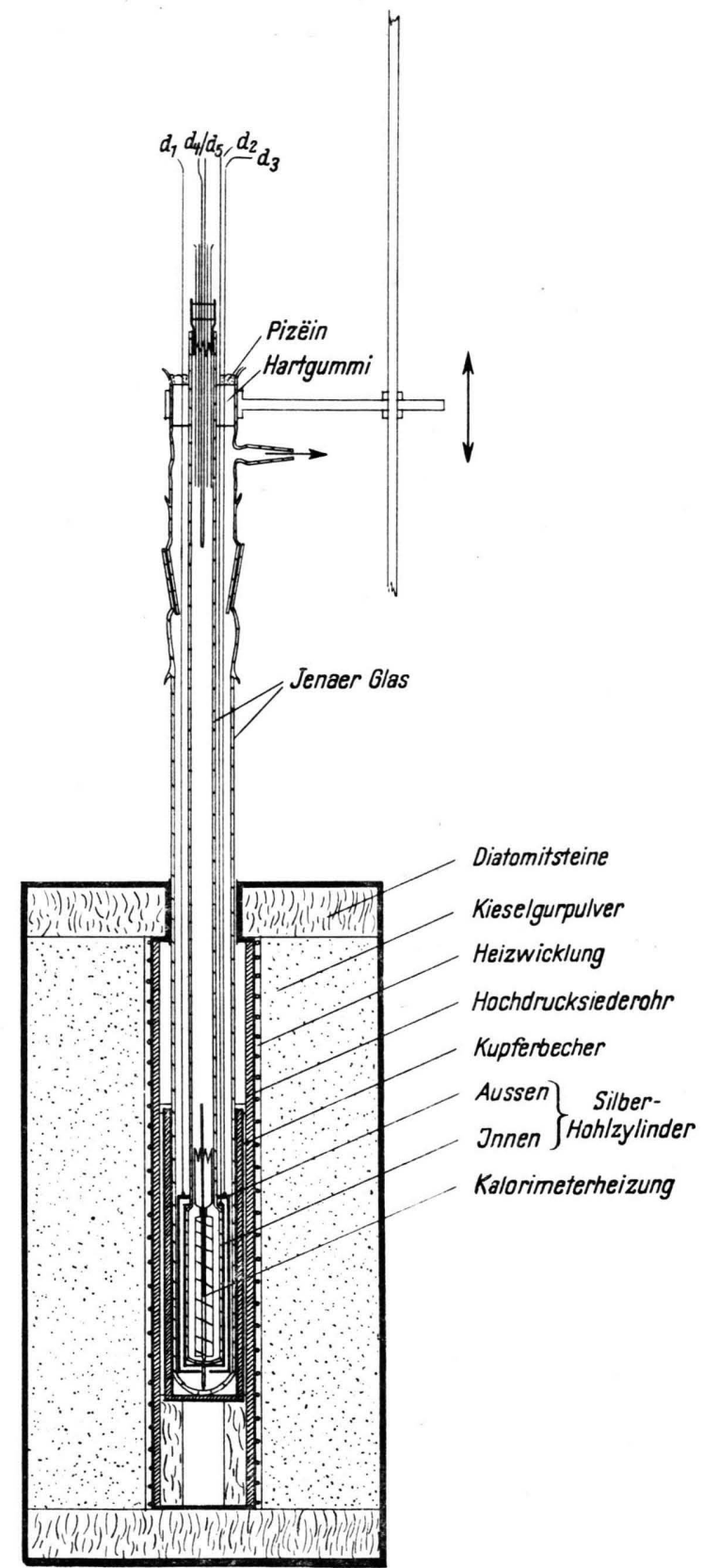

Abb. 1. Adiabatisches Kalorimeter (Gesamtan sicht)

7 M. P. Mondain-Monval, Bull. Soc. Chim. France (4) 39, 1349 [1926]; M. P. Mondain-Monval u. P. Schneider, ebd. (4) 43, 1302 [1928]; M. P. Mondain-Monval, R. Job u.P. Galet, ebd. (4) 47, 545 [1930].

${ }^{8}$ H. Moser, Phys. Z. 37, 737 [1936]. 
$180^{\circ}$ zueinander symmetrisch angeordnete Lötstellen. Von diesen führen (insgesamt fünf) längs der Wandungen in Quarzkapillaren isoliert verlegte gleich lange Konstantandrähte $(0,4 \mathrm{~mm} \varnothing) \mathrm{zu}$ einer gemeinsamen Lötstelle dicht oberhalb des äußeren Silberzylinders. Von dieser Lötstelle setzt sich ein einziger Konstantandraht $d_{1}$ nach oben fort. Ebenso führen von den Oberseiten beider Silberhohlzylinder je ein Silberdraht $(0,25 \mathrm{~mm} \varnothing)$ gleichen Materials (keine feststellbare unterschiedliche Thermokraft!) $d_{2}$ und $d_{3}$ nach oben, weiter gemeinsam mit dem Konstantandraht $d_{1}$ durch Bohrungen eines am Kopfe befindlichen Hartgummistopfens und durch einen Pizëinvergu $B$ vakuumdicht ins Freie. Sie sind im Innern wie außerhalb der Apparatur durch Glaskapillaren vor gegenseitiger Berührung geschützt.

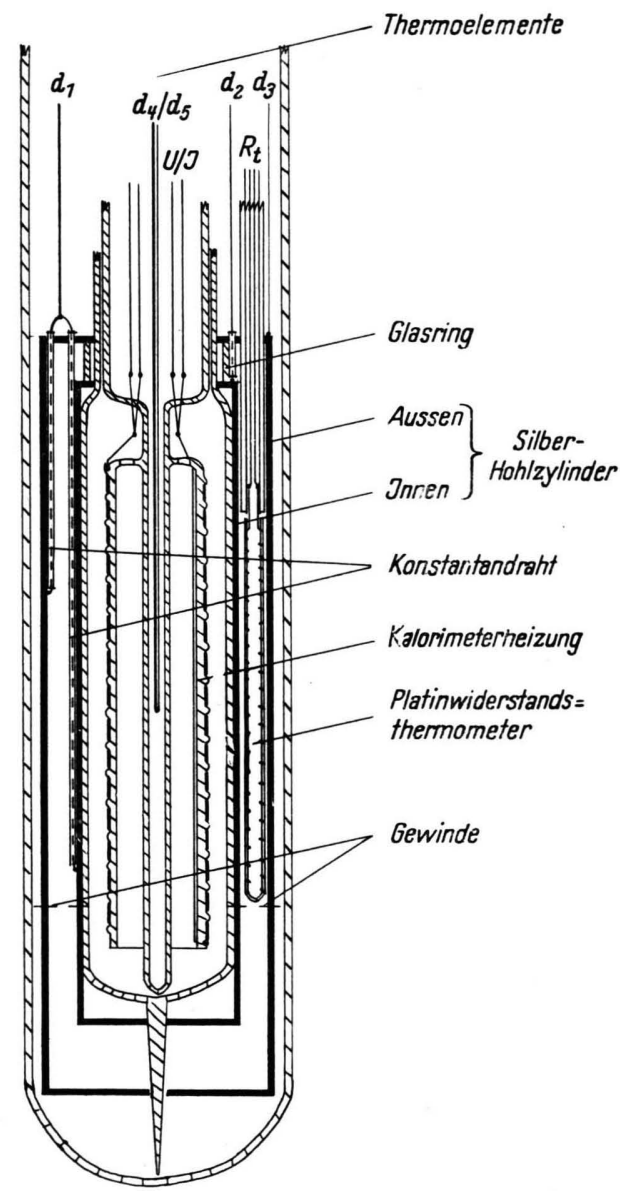

Abb. 2. Kalorimetereinsatz mit Heizer, Widerstandsthermometer und Thermoelementanordnung.

Der ebenfalls aus Glas gefertigte Heizer steht unten auf dem Boden des Kalorimeters auf. Die Heizwicklung aus $1 \mathrm{~mm}$ breitem Platinband (0,05 mm stark) ist in eine mittels $70 \%$ iger Flußsäure eingeätzte Rillung verlegt. Vom Heizwiderstand $(6,481 \mathrm{Ohm}$ bei $0^{0} \mathrm{C}$ ) führen zwei Platindrahtdurchführungen und in
Fortsetzung dieser je zwei Drähte durch den Hohlschaft des Heizers nach oben ins Freie.

Wie aus Abb. 2 weiter zu ersehen, ist im Mittelpunkt des Kalorimeters zur Messung der Achsentemperatur $\left(t_{1}\right)$ ein Silberkonstantan-Thermoelement $\left(d_{4} \ldots d_{5}\right)$ angeordnet, sowie zwischen beiden Silberhohlzylindern zwecks Eichung der Thermoelementkombination ein Platinwiderstandsthermometer mit vier Silberdrahtzuführungen untergebracht, die in einem Glasrohr (isoliert) nach oben durch den Hartgummizylinder vakuumdicht ins Freie laufen. Die freien Enden der Thermodrähte $d_{1}, d_{2}, d_{3}, d_{4}$ und $d_{5}$ enden mit Lötstellen in engen, mit Öl gefüllten Glasröhrchen, die durch Bohrungen eines Gummistopfens in ein Dewargefä $B$ $\left(0^{0} \mathrm{C}\right)$ führen. Von diesen Lötstellen verlaufen Kupferleitungen $\mathrm{zu}$ einem thermokraftfreien Quecksilberumschalter und -polwender.

\section{Meßverfahren}

Zur Eichung des Kalorimeters und zur Bestimmung der wahren spezifischen Wärme hat sich folgendes adiabatische Meßverfahren bewährt: Während die Temperatur des Ofens mit sehr geringer, nahezu unveränderter Geschwindigkeit ansteigt, wird die dem Kalorimeter zugeführte elektrische Leistung so einreguliert, daß die mit dem Thermoelement $d_{2} \ldots d_{3}$ $\left(t_{3}-t_{2}=\Delta \vartheta\right)$ festzustellende Temperaturdifferenz zwischen Kalorimeteroberfläche $\mathrm{d}_{1} \ldots \mathrm{d}_{2}\left(t_{2}\right)$ und Umgebung $d_{1} \ldots d_{3}\left(t_{3}\right)$ nach Möglichkeit Null wird. Sodann kann bei konstanter Kalorimeterleistung die Folge einer Meßreihe begonnen werden. Es wird jeweils für eine Einzelmessung die Zeit $\Delta z$ beobachtet, während der ein bestimmter, nie größer als $1^{0} \mathrm{C}$ gewählter Temperaturanstieg des Kalorimeters $\Delta t$ stattfindet. Sowohl der durch das Thermoelement $d_{1} \ldots d_{2}$ $\left(t_{2}\right)$ feststellbare Temperaturanstieg des Kalorimeters an der Oberfläche $\left(\Delta t_{2}\right)$ als auch der durch das Thermoelement $d_{4} \ldots d_{5}\left(t_{1}\right)$ wahrzunehmende Anstieg der Achsentemperatur $\left(\Delta t_{1}\right)$ des Kalorimeters werden mit Hilfe eines Dießelhorst-Kompensators gemessen und durch zwei bestimmte Einstellungen dieses Apparates (Werte der EMK) im voraus festgelegt. Dabei werden jeweils die Zeiten $\Delta z_{1}$ und $\Delta z_{2}$ als die Zeitdauer zwischen den beiden Durchgängen der Lichtmarke durch den Nullpunkt der Galvanometerskala bestimmt. Zwischen beiden Temperaturanstiegen $\left(\Delta t_{1}, \Delta t_{2}\right)$ und den zugehörigen Zeiten $\left(\Delta z_{1}, \Delta z_{2}\right)$ wird arithmetisch gemittelt, um dem örtlich unterschiedlichen Temperaturgang des Kalorimeters und der Versuchssubstanz möglichst Rechnung zu tragen. Das bei den Messungen vorliegende Temperaturgefälle zwischen Temperatur der Kalorimeterheizung $\left(t_{\mathrm{H}}\right)$ einerseits und Oberflächentemperatur $\left(t_{2}\right)$ bzw. der Achsentemperatur $\left(t_{1}\right)$ andererseits betrug je nach Höhe der dem Temperaturgang entsprechenden Heizleistung des Kalorimeters nur etwa 2 bis $3^{0} \mathrm{C}\left(t_{\mathrm{H}}-t_{2}\right)$ bzw. 1 bis $2^{0} \mathrm{C}\left(t_{\mathrm{H}}-t_{1}\right)$.

Die Adiabasie zwischen Kalorimeter und Umgebung wird im allgemeinen nicht ganz erreicht; daher wurde der Wärmeaustausch zwischen Kalorimeter und Umgebung durch 


$$
k_{(t)}=k_{1(t)} \Delta \vartheta-k_{2(t)}
$$

berücksichtigt. Hierin bedeutet $k_{(t)}$ die aus der Umgebung pro Zeiteinheit zugeführte Wärme; $k_{1(t)} \Delta \vartheta$ die vom äußeren Silberhohlzylinder her zufließende Wärme, wenn dessen Temperatur $\left(t_{3}\right)$ um $\Delta \vartheta$ größer als $t_{2} ; k_{2(t)}$ den Wärmeverlust durch Ableitung nach oben. $k_{2(t)}$ wird bestimmt durch Messung der Kalorimeterleistung, die erforderlich ist, um bei verschwindendem Wärmeaustausch nach der Seite $(\Delta \vartheta=0)$ eine konstante Kalorimetertemperatur zu erzielen; $k_{1(t)}$ wird aus zwei mit gleichem Temperaturgang verlaufenden Meßreihen A und B erhalten, von denen die erste eine Temperaturdifferenz $\Delta \vartheta=0$, die andere stets eine solche von etwa $\Delta \vartheta=+2^{0} \mathrm{C}$ im gesamten Verlauf innehält. In der und Spannung $U$ an den Enden des Heizwiderstandes.

Die Wärmekapazität des leeren Kalorimeters (Wasserwert) $W t$ bzw. die wahre spezifische Wärme bei konstantem Druck des flüssigen Schwefels $c t$ ergeben sich aus den Gleichungen

$W_{t} \Delta t=0,239\left(U I_{(t)}+k_{1(t)} \Delta \vartheta-k_{2(t)}\right) \Delta z$

$g c_{t} \Delta t=0,239\left(U I_{(t)}+k_{1(t)} \Delta \vartheta-k_{2(l)}\right) \Delta z-W_{t} \Delta t$

in cal/Grad. Hierin bedeutet $g$ die Masse des eingewogenen Schwefels in Gramm, $c_{t}$ die wahre spezifische Wärme desselben bei $t^{0} \mathrm{C}$. Die so bestimmte wahre spezifische Wärme bezieht sich auf die mittlere Temperatur des Schwefels, die man in guter Näherung durch Mittelbildung aus den während

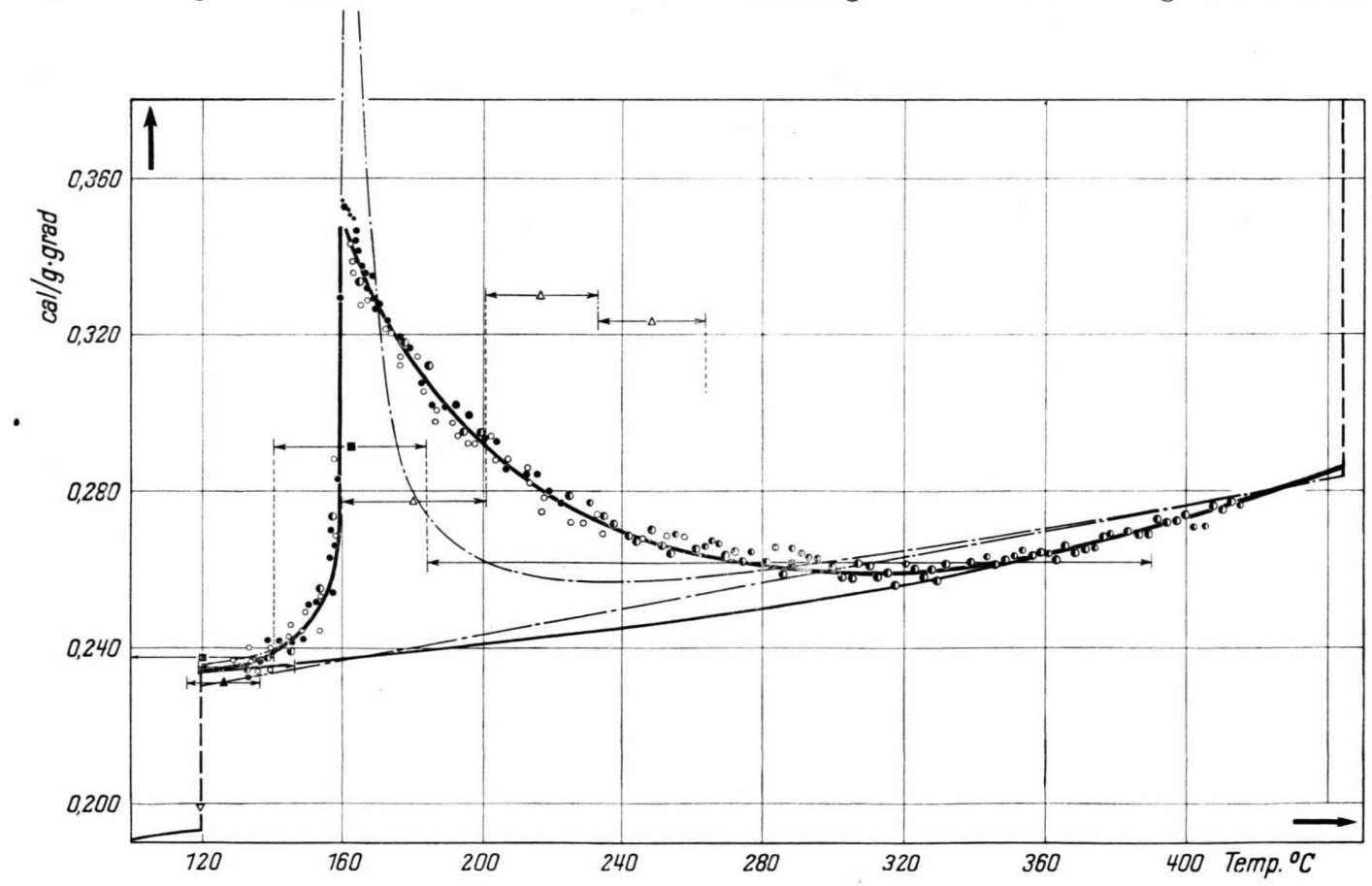

Abb. 3. Die wahre spezifische Wärme des flüssigen Schwefels $c_{t}$. Mittlere spez. Wärme nach Person $\nabla$, nach Classen $\Delta$, nach Jitaka $\nabla$, nach Dussy $\triangle$, nach Lewis u. Randall $\mathbf{\square}, \cdot-\cdots-$ wahre spez. Wärme, - - - - , ,normale“" spez. Wärme nach Lewis u. R andall. Meßreihe 1, $2: \bigcirc$ (Temp.-Gang: 0,290 C/min); Meßreihe 3, 4, 5: $\left(0,24^{\circ} \mathrm{C} / \mathrm{min}\right)$; Meßreihe 6, $7: \bigcirc\left(0,15^{0} \mathrm{C} / \mathrm{min}\right) . \longrightarrow$ wahre spez. Wärme $c_{t}$ nach dieser Arbeit, $\longrightarrow$,normale“ spez. Wärme $c_{t \text { (norm.) }}$

Meßreihe B wird daher eine um die Wärmeaufnahme $k_{1(t)} \Delta \vartheta$ geringere Kalorimeterleistung $(U I)_{\mathrm{B}}$ als bei Meßreihe A gemessen. Die Temperaturdifferenz $\Delta \vartheta$, die sich bei allen Messungen zur Eichung und Bestimmung der wahren spezifischen Wärme zwischen ca. $+1^{0}$ und $-1^{0}$ bewegte, wird während einer Messung etwa dreimal auf einige Tausendstel Grad genau bestimmt, ebenso oft die Stromstärke $I$ einer Messung zeitlich gemittelten Temperaturwerten $t_{1}, t_{2}$ und $t_{\mathrm{H}}$ erhält; diese war bei kleinen Kalorimeterleistungen praktisch mit $t_{1}$ identisch.

Die beiden durch Gl. (1) definierten Korrekturposten in (3) hatten bei den Messungen zusammen eine Größe von ca. 10 bis $15 \%$ des Hauptgliedes, während die Wärmekapazität des leeren Kalorimeters mit etwa $30 \%$ beteiligt war. Die Fehlerrech- 
nung ergab einen mittleren Gesamtfehler für jede Einzelbestimmung von etwa $\pm 1,5 \%$.

Der Schwefel wurde in Toluol umkristallisiert, zweimal im Vakuum sublimiert und 125,0 g gepulverte Substanz in das Kalorimeter eingebracht, das im geschmolzenen Zustand des Schwefels fast bis zum Halsansatz gefüllt war. Bei 3 ausgesuchten, nahezu konstanten Temperaturgängen des Kalorimeters mit einem durchschnittlichen Temperaturanstieg von $0,29,0,24$ und $0,15^{\circ} \mathrm{C} / \mathrm{min}$ wurden 7 Meßreihen mit insgesamt 168 Einzelbestimmungen der wahren spezifischen Wärme des flüssigen Schwefels zwischen 130 und $420^{\circ} \mathrm{C}$ durchgeführt.

\section{Ergebnisse und Diskussion}

Die Meßergebnisse sind in Abb. 3 aufgetragen, wobei die zu den drei angewandten Temperaturgängen des Kalorimeters gehörenden Werte gekennzeichnet sind. Wie man sieht, treten nirgends systematische Abweichungen auf, wie man sie er-

\begin{tabular}{|c|c|c|c|}
\hline $\begin{array}{c}\text { Temp. } \\
\left({ }^{\circ} \mathrm{C}\right)\end{array}$ & $c_{t}$ & $c_{t(\text { norm. })}$ & $\begin{array}{c}\left(c_{t}-c_{t} \text { (norm.) }\right) \\
\cdot 10^{3}\end{array}$ \\
\hline 120 & $(0,234)$ & $(0,234)$ & 0 \\
\hline 30 & 0,236 & 234 & 2 \\
\hline 40 & 239 & 235 & 4 \\
\hline 50 & 0,246 & 0,236 & 7 \\
\hline 60 & 0,347 & 237 & 110 \\
\hline 70 & 328 & 238 & 90 \\
\hline 80 & 314 & 239 & 75 \\
\hline 90 & 302 & 240 & 62 \\
\hline 200 & 0,292 & 0,241 & 51 \\
\hline 10 & 285 & 242 & 43 \\
\hline 20 & 279 & 243 & 36 \\
\hline 30 & 274 & 244 & 30 \\
\hline 40 & 270 & 245 & 25 \\
\hline 50 & 0,267 & 0,246 & 21 \\
\hline 60 & 264 & 247 & 17 \\
\hline 70 & 262 & 248 & 14 \\
\hline 80 & 261 & 250 & 11 \\
\hline 90 & 260 & 251 & 9 \\
\hline 300 & 0,259 & 0,253 & 7 \\
\hline 10 & 259 & 254 & 5 \\
\hline 20 & 259 & 256 & 3 \\
\hline 30 & 260 & 258 & 2 \\
\hline 40 & 261 & 260 & 1 \\
\hline 50 & 0,262 & 0,261 & 1 \\
\hline 60 & 264 & 263 & 1 \\
\hline 70 & 266 & 266 & 一 \\
\hline 80 & 268 & 268 & - \\
\hline 90 & 270 & 270 & - \\
\hline 400 & 0,273 & 0,273 & 一 \\
\hline 10 & 276 & 276 & 一 \\
\hline 20 & 279 & 279 & 一 \\
\hline 30 & $(0,282)$ & $(0,282)$ & - \\
\hline 40 & $(0,285)$ & $(0,285)$ & - \\
\hline
\end{tabular}

Tab. 1. Die wahre spezifische Wärme des flüssigen Schwefels. warten müßte, wenn die Einstellung des Gleichgewichts im Schwefel dem Temperaturanstieg nicht folgen würde. Die aus der ausgeglichenen Kurve entnommenen Werte sind in Tab. 1 für eine Anzahl Temperaturen zwischen 120 und $440^{\circ} \mathrm{C}$ mit einer Genauigkeit von etwa $\pm 0,5 \%$ angegeben. Dies dürfte auch die Genauigkeit des Ergebnisses darstellen, abgesehen vom Bereich zwischen 150 und $170^{\circ}$, wo wir wegen der starken Temperaturabhängigkeit der spezifischen Wärme die Fehlergrenze auf etwa $1 \%$ schätzen. Des weiteren sind in Spalte 3

\begin{tabular}{|c|c|c|c|}
\hline Temp. $\left({ }^{0} \mathrm{C}\right)$ & $H_{t}-32$ & $H_{t(\text { norm. })}-32$ & $H_{t}-H_{t \text { (norm.) }}$ \\
\hline 120 & 0 & 0 & - \\
\hline 30 & 2,35 & 2,34 & 0,01 \\
\hline 40 & 4,72 & 4,70 & 0,02 \\
\hline 50 & 7,15 & 7,07 & 0,08 \\
\hline 60 & 9,80 & 9,44 & 0,36 \\
\hline 65 & 11,5 & 10,6 & 0,95 \\
\hline 70 & 13,3 & 11,8 & 1,45 \\
\hline 75 & 14,9 & 13,0 & 1,88 \\
\hline 80 & 16,4 & 14,2 & 2,26 \\
\hline 85 & 17,9 & 15,3 & 2,59 \\
\hline 90 & 19,4 & 16,5 & 2,89 \\
\hline 200 & 22,4 & 18,9 & 3,48 \\
\hline 10 & 25,3 & 21,3 & 3,98 \\
\hline 20 & 28,1 & 23,7 & 4,37 \\
\hline 30 & 30,8 & 26,1 & 4,68 \\
\hline 40 & 33,5 & 28,5 & 4,95 \\
\hline 50 & 36,2 & 31,0 & 5,20 \\
\hline 60 & 38,9 & 33,4 & 5,44 \\
\hline 70 & 41,5 & 35,9 & 5,62 \\
\hline 80 & 44,1 & 38,4 & 5,74 \\
\hline 90 & 46,6 & 40,8 & 5,81 \\
\hline 300 & 49,2 & 43,3 & 5,88 \\
\hline 10 & 51,8 & 45,8 & 5,94 \\
\hline 20 & 54,4 & 48,4 & 5,98 \\
\hline 30 & 56,9 & 50,9 & 6,02 \\
\hline 40 & 59,5 & 53,5 & 6,06 \\
\hline 50 & 62,1 & 56,0 & 6,09 \\
\hline 60 & 64,7 & 58,6 & 6,11 \\
\hline 70 & 67,4 & 61,4 & 6,13 \\
\hline 80 & 70,1 & 64,0 & 6,14 \\
\hline 90 & 72,9 & 66,7 & 6,15 \\
\hline 400 & 75,7 & 69,6 & 6,16 \\
\hline 10 & 78,4 & 72,3 & 6,16 \\
\hline 20 & 81,2 & 74,9 & 6,16 \\
\hline 30 & $(83,9)$ & $(77,7)$ & $(6,16)$ \\
\hline 40 & $(86,7)$ & $(80,5)$ & $(6,16)$ \\
\hline
\end{tabular}

Tab. 2. Der Wärmeinhalt des flüssigen Schwefels bezogen auf $H_{15^{\mathrm{n}}}=0, H_{120^{\circ}}=32$.

derselben Tabelle die Werte für die ,normale“ spezifische Wärme des flüssigen Schwefels $c_{t \text { (norm.), }}$ worunter hier die spezifische Wärme ohne Berücksichtigung der Anomalie verstanden wird, angegeben. Für den wahrscheinlichsten Verlauf dieser „,normalen" spezifischen Wärme wurde eine quadratische Funktion $c_{t \text { (norm.) }}=a+b t+c t^{2}$ angesetzt 
(siehe Abb. 3), deren Konstanten so bestimmt wurden, daß die gemessenen Werte bei $120^{\circ} \mathrm{C}$ und oberhalb des Anomalitätsgebietes (etwa ab $350^{\circ} \mathrm{C}$ aufwärts) wiedergegeben wurden. In Spalte 4 der Tab. 1 sind schließlich die sich aus den Differenzen der wahren und ,normalen“ spezifischen Wärme des flüssigen Schwefels ergebenden differentiellen Umwandlungswärmen verzeichnet.

Der Wärmeinhalt des flüssigen Schwefels $H_{t}$ zwischen 120 und $440^{\circ} \mathrm{C}$ wurde durch graphische Integration der wahren spezifischen Wärme $c_{t}$ ermittelt und ist in Tab. 2 angegeben. Darin sind die Wärmeinhalte $H_{t}$ auf $H_{15^{\circ} \mathrm{C}}=0$ bezogen. Der so definierte Wärmeinhalt bei $120^{\circ} \mathrm{C}, H_{120}$, ergibt sich zu $32 \mathrm{cal} / \mathrm{g}$ und dem Verdampfungspunkt, $H_{444,6}$, erhält man somit rund $120 \mathrm{cal} / \mathrm{g}$. Wie Abb. 4 zeigt, stimmen die von Lewis und Randall ${ }^{5}$ sowie MondainMonval ${ }^{7}$ erzielten Meßergebnisse innerhalb der von ihnen erreichten Meßgenauigkeit mit dem von uns gewonnenen Verlauf des Wärmeinhaltes $H_{t}$ (bezogen auf $15^{\circ} \mathrm{C}$ ) leidlich überein.

Auf die von Mondain-Monval gefolgerten Angaben über die spezifische Wärme braucht nicht näher eingegangen zu werden, da er von der sicher unzutreffenden Voraussetzung eines (echten) Umwandlungspunktes bei $160^{\circ} \mathrm{C}$ ausgeht. Lew is und $\mathrm{R}$ andall haben aus ihren Wärmeinhaltsmessungen unter der Annahme des von Smith ${ }^{6}$ und Mitarbei-

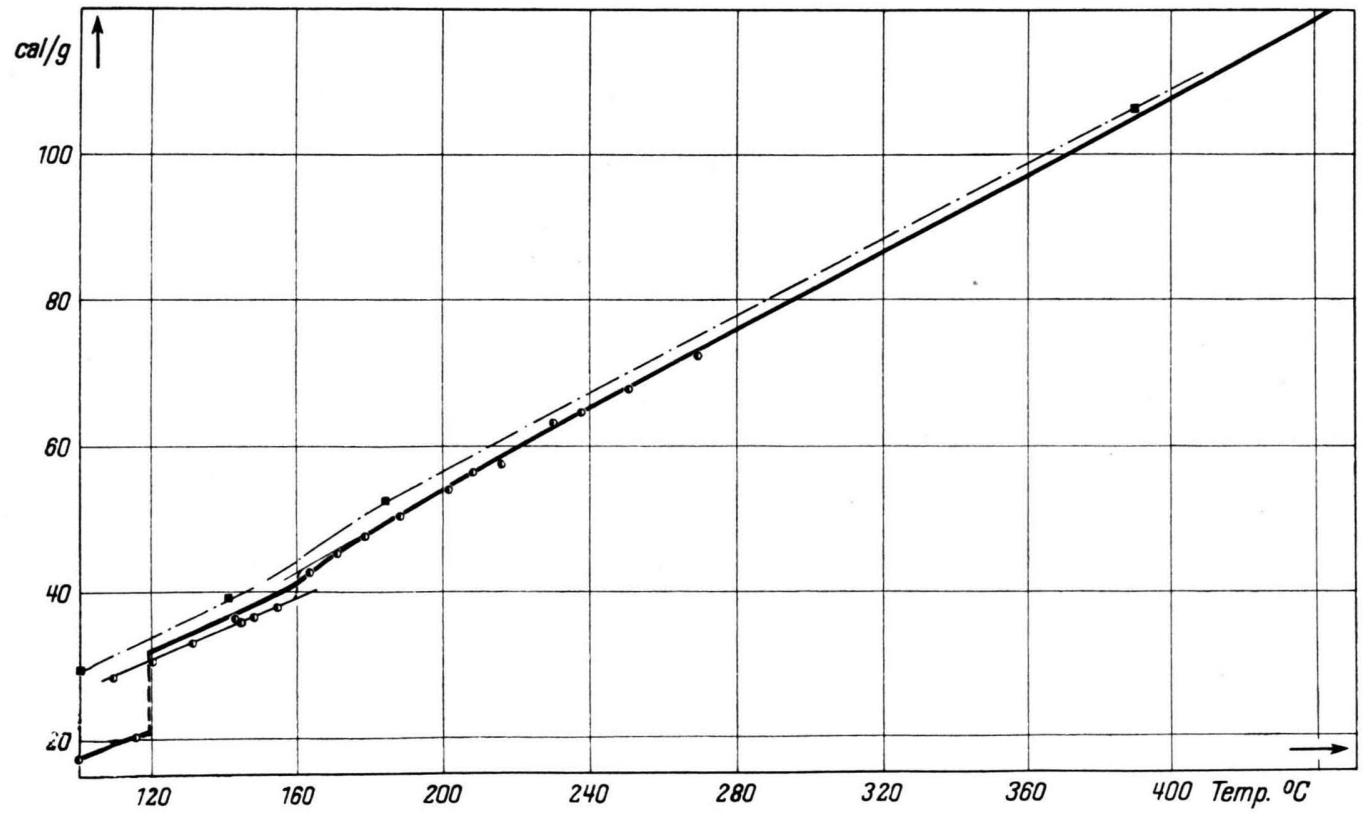

Abb. 4. Der Wärmeinhalt des festen und flüssigen Schwefels $H_{t}-H_{15}$. Messungen von Lewis u. Randall $\square$, von Mondain-Monval D; Wärmeinhalt $H_{t}$ nach Mondain-Monval—, nach dieser Arbeit —

unter Benutzung der von Eastman und McGav o ck ${ }^{9}$ bestimmten wahren spezifischen Wärmen des festen Schwefels sowie Berücksichtigung einer Umwandlungswärme $S_{\mathrm{rh}} \rightarrow S_{\mathrm{m}}$ von $2,8 \mathrm{cal} / \mathrm{g}$ und einer Schmelzwärme des $S_{\text {mon }}$ von $10,5 \mathrm{cal} / \mathrm{g}$. Entsprechend wurde der ,,normale“"Wärmeinhalt (Spalte 3) und durch Differenzbildung die Umwandlungswärme zwischen 120 und $t^{0} \mathrm{C}$ (Spalte 4 ) berechnet. Für den Wärmeinhalt des Schwefels zwischen $15^{\circ} \mathrm{C}$

9 E. D. Eastman u. W. C. Mc Gavock, J. Amer. Chem. Soc. 59, 145 [1937]. tern bestimmten temperaturabhängigen Gleichgewichtes $S_{\lambda} \rightleftarrows S_{\mu}$ den Verlauf der wahren spezifischen Wärme des flüssigen Schwefels zu berechnen versucht; die so erhaltenen Werte stehen jedoch mit unseren direkten Messungen der wahren spezifischen Wärme in Widerspruch (siehe Abb. 3). Wir haben nun umgekehrt auf Grund unserer Meßergebnisse auf den Verlauf der Gleichgewichtskurve für das thermodynamische Gleichgewicht $S_{\lambda} \rightleftarrows S_{\mu}$ zu schließen versucht. Unter der auch von Lew is und Randall zugrunde gelegten Annahme eines einfachen thermodynamischen Gleichgewich- 
tes lassen sich dann die wahre spezifische Wärme bzw. der Wärmeinhalt des flüssigen Schwefels darstellen durch

$$
\begin{gathered}
c_{t}=c_{t(\text { norm. })}+q\left(\frac{\mathrm{d}(y / 100)}{\mathrm{d} t}\right), \\
\left(H_{t}-32\right)=\left(H_{t(\text { norm. })}-32\right)+q \int_{120}^{t}\left(\frac{\mathrm{d}(y / 100)}{\mathrm{d} t}\right) d t .
\end{gathered}
$$

Hierin bedeutet $q$ die als temperaturunabhängig angenommene Umwandlungswärme pro Gramm S und $y_{t}$ der bei der Temperatur $t$ vorhandene Anteil unlöslichen Schwefels in Gewichtsprozenten. Nach den in Tab. 3, Spalte 2 bis 5, enthaltenen Angaben über den unlöslichen Schwefel nach $\mathrm{Smith}^{6}$ und Mitarbb., Hammick, Cousins und Langford ${ }^{10}$, Powell und Eyring ${ }^{11}$ sowie Gee ${ }^{12}$ wurden die in Abb. 5 wiedergegebenen Gleichgewichtskurven gezeichnet. Es ist zu bemerken, daß die Angaben von Powell und Eyring sowie von Gee nicht auf Abschreckversuchen beruhen, sondern das Ergebnis von theoretischen Berechnungen sind, deren Grundlage die Annahme ist, daß der unlösliche Schwefel ein Polymerisat mit Ketten variabler Länge dar-

${ }^{10}$ D. L. Hammick, W. R. Cousins u. W. E. J. Langford, J. Chem. Soc. 1928, 797. stellt; die Voraussetzung ist daher eine etwas

\begin{tabular}{|c|c|c|c|c|c|}
\hline \multirow[t]{2}{*}{${ }^{\text {Temp }} \mathrm{C}}$. & \multicolumn{4}{|c|}{$\begin{array}{c}y_{t} \\
\text { nach verschiedenen } \\
\text { Autoren }\end{array}$} & \multirow[t]{2}{*}{$\begin{array}{c}y_{t} \\
\text { berechn. }\end{array}$} \\
\hline & 6 & 10 & 11 & 12 & \\
\hline 120 & 3,6 & - & 0,7 & - & 6,8 \\
\hline 30 & 4,4 & - & 1,7 & - & 6,9 \\
\hline 40 & 5,6 & $(2,3)$ & 3,7 & 一 & 7,0 \\
\hline 50 & 7,4 & 4,2 & 7,2 & - & 7,5 \\
\hline 60 & 11,0 & 9,1 & 12,5 & 0 & 9,8 \\
\hline 65 & 15,1 & 14,2 & 15,8 & - & 14,7 \\
\hline 70 & 19,3 & 18,4 & 19,0 & 11,0 & $18 ; 9$ \\
\hline 75 & 22,0 & 22,4 & 22,3 & - & 22,5 \\
\hline 80 & 23,4 & 26,0 & 25,6 & 19,9 & 25,6 \\
\hline 85 & 24,8 & 29,1 & 28,5 & - & 28,4 \\
\hline 90 & 25,6 & 32,4 & 31,4 & 26,5 & 30,9 \\
\hline 200 & 27,1 & 36,3 & 35,9 & 32,7 & 35,8 \\
\hline 10 & 28,2 & 38,1 & 39,4 & 38,4 & 40,0 \\
\hline 20 & 29,1 & 38,8 & 42,0 & 43,5 & 43,2 \\
\hline 40 & 30,5 & 39,2 & 45,3 & 52,2 & 48,1 \\
\hline 60 & 31,5 & 39,3 & 47,2 & 58,8 & 52,1 \\
\hline 80 & 32,3 & 39,4 & 48,2 & 63,6 & 54,6 \\
\hline 300 & 32,8 & 39,4 & 48,9 & 67,7 & 55,8 \\
\hline 20 & 33,2 & 39,4 & 49,2 & 71,2 & 56,6 \\
\hline 60 & 33,9 & 39,4 & 49,6 & 76,8 & 57,7 \\
\hline 400 & 34,3 & 39,4 & 49,8 & 81,3 & 58,0 \\
\hline 40 & 34,5 & 39,4 & 50,0 & 84,4 & 58,2 \\
\hline
\end{tabular}
andere als die oben gemachte eines Gleichgewichts

Tab. 3. Gew.-Proz. $\left(y_{t}\right)$ unlöslichen Schwefels.

${ }^{11}$ R. E. Powell u. H. Eyring, J. Amer. Chem. Soc. 65, 648 [1943].

12 G. Gee, Trans. Faraday Soc. 48, 515 [1952].

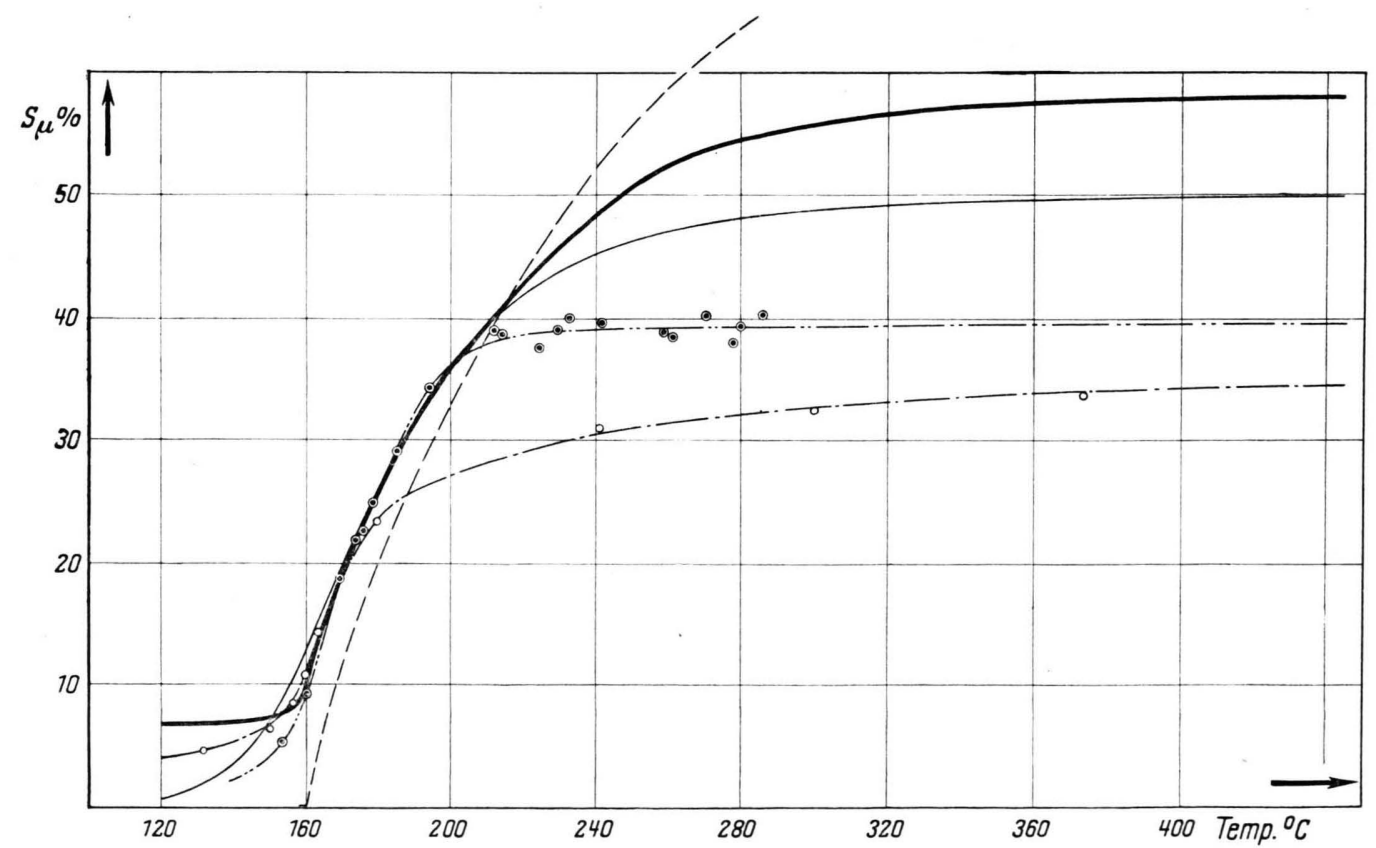

Abb. 5. Gew.-Proz. unlöslichen Schwefels im flüssigen Schwefel. Gleichgewichtskurve nach Smith u. Holmes

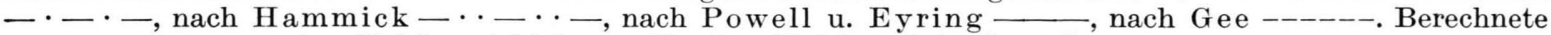
Gleichgewichtskurve für das Gleichgewicht $\mathbf{S}_{\gamma} \rightleftarrows \mathbf{S}_{\mu}$ 
zwischen $S_{\lambda}$ und einem einheitlichen chemischen Individuum $S_{\mu}$. Da alle Gleichgewichtskurven mit Ausnahme der von Gee, deren theoretische Begründung jedoch kaum anerkannt werden kann, in dem Bereich von $165-185^{\circ}$ praktisch zusammenfallen, wurden die in diesem Bereich am wahrscheinlichsten gültigen Werte für $y_{t}$ der Berechnung von $q$ zugrunde gelegt. Aus den Differenzbeträgen der Umwandlungswärmen einerseits und den zugehörigen Änderungen des Gehaltes an unlöslichem Schwefel erhielten wir gemäß der Gleichung

$$
\begin{aligned}
{\left[H_{t}-H_{t(\text { norm. })}\right]_{2}-[} & \left.H_{t}-H_{t(\text { norm. })}\right]_{1} \\
& =q \int_{t_{1}}^{t_{2}}\left(\frac{\mathrm{d}(y / 100)}{\mathrm{d} t}\right) d t
\end{aligned}
$$

als Mittelwert $q=12,0 \pm 0,7 \mathrm{cal} / \mathrm{g}$. Mit diesem Wert wurde sodann, ausgehend von $170^{\circ} \mathrm{C}$ als Bezugspunkt (1,45 cal Umwandlungswärme und 18,9\% $S_{\mu}$ ) nach niedrigerer und höherer Temperatur hin der gesamte Verlauf der Gleichgewichtskurve berechnet, die mit allen Gleichgewichtskurven in erheblichem Widerspruch steht; die größte Diskrepanz besteht im hohen Temperaturbereich bis $440^{\circ} \mathrm{C}$, wo rund $58 \% S_{\mu}$ zu erwarten wären, während Smith nur etwa $34 \%$ und Hammick ca. $40 \%$ findet.

Abb. 5 zeigt, daß die Abschreckversuche von Smith sowie Hammick besonders bei höheren Temperaturen ganz bedeutend niedrigere Prozentgehalte an unlöslichem Schwefel ergaben als nach unserer Rechnung zu erwarten wäre; sie weichen jedoch auch voneinander stark ab. Die Schwierigkeit der Versuche besteht darin, daß bei höheren Temperaturen wegen der großen Reaktionsgeschwindigkeit kaum ein ideales Einfrieren des Gleichgewichts zu garantieren ist. So hatte denn auch die wesentliche Verbesserung der Abschreckmethode durch $\mathrm{Ham}$ - mick ein erhebliches Anwachsen der Werte bei höheren Temperaturen im Vergleich mit Smith zur Folge. Die wirklichen Gleichgewichtswerte liegen möglicherweise noch höher als die von Hammick angegebenen.

Wenn man also in dem Ergebnis der Abschreckversuche auch nicht unbedingt einen Widerspruch zu dem der obigen Rechnung zu sehen braucht, so ist doch die zugrunde liegende Annahme eines Gleichgewichtes $S_{\lambda} \rightleftarrows S_{\mu}$, wobei unter $S_{\mu}$ ein einheitliches chemisches Individuum verstanden ist, sehr unwahrscheinlich. Vielmehr wird man zu einer befriedigenden Interpretation wohl eher kommen, wenn man berücksichtigt, daß aus den Beobachtungen über die Viskosität von Schwefelschmelzen mit Sicherheit auf die Gegenwart von mit steigender Temperatur wachsenden Mengen von polymerisierten Molekülen im Gleichgewicht geschlossen werden kann, wobei anzunehmen ist, daß die durchschnittliche Kettenlänge eine Funktion der Temperatur ist. Es liegt nahe, den unlöslichen Schwefel mit den polymeren Molekülen zu identifizieren.

Powell und Eyring haben, wie bereits oben erwähnt, das Problem von diesem Standpunkt aus behandelt. Das Ergebnis ist insofern nicht ganz befriedigend, als nach ihrer Theorie zwar ein starkes Anwachsen der Viskosität, aber doch nicht das fast sprungartig steile Ansteigen bei $160^{\circ}$ folgt, wie es tatsächlich der Fall ist; sie finden als Grenzwert des Gehaltes an unlöslichem Schwefel $50 \%$, während Hammick und Mitarbb. etwa $40 \%$ fanden. Auch die Behandlung des Problems durch Gee ist starken Einwänden ausgesetzt. Wir halten den Versuch einer theoretischen Interpretation der Messungen nicht für sehr lohnend, bevor weiteres experimentelles Material über den inneren Zustand der Schwefelschmelzen vorliegt. 